\title{
Evaluation of Lupus Low Disease Activity: Characteristics, Predictors, and Association with Disease Damage: A Retrospective Cohort from Two Tertiary Centers in Egypt
}

\author{
BASMA M. MEDHAT, M.D.*; MERVAT E. BEHIRY, M.D.** and NAGLAA AFIFI, M.D.** \\ The Department of Rheumatology \& Rehabilitation* and Internal Medicine Department**, Faculty of Medicine, Cairo University, \\ Cairo, Egypt
}

\begin{abstract}
Background: Remission in systemic lupus erythematosus (SLE) is seldom achieved; making Lupus Low Disease Activity (LLDA) an alternative yet promising target.

Aim of Study: The aim of this study was to evaluate the prevalence of remission and LLDA achieved, and the characteristics and predictors of LLDA, and its potential association with disease damage.

Patients and Methods: The medical records of 243 patients fulfilling the 2012 Systemic Lupus Collaborating Clinics (SLICC) classification criteria for SLE and managed at Cairo and Ainshams Universities from January to December 2019 were viewed. Remission was categorized to: (i) Complete remission off glucocorticoid (GC) and Systemic Lupus Erythematosus Disease Activity Index-2K (SLEDAI-2K) = zero (antimalarial only); (ii) Clinical remission off GC with serologic activity (SKLEDAI-2K $\leq 4$ ); (iii) Clinical remission on GC $\leq 5 \mathrm{mg}$ /day (SLEDAI-2K score $\leq 4$ and serologic activity). LLDA was defined as SLEDAI-2K $\leq 4$ in the absence of major organ involvement, GC dosage $\leq 7.5 \mathrm{mg} /$ day. Disease damage was assessed through the Systemic Lupus International Collaborating Clinics/American College of Rheumatology Damage Index (SDI).

Results: Seventy two (29.6\%) patients achieved LLDA at the last visit; whereas $142(58.4 \%)$ patients had a SLEDAI$2 \mathrm{~K}$ score $>4$ and/or received a GC dosage of $>7.5 \mathrm{mg}$. One $(0.4 \%)$ and $28(11.5 \%)$ patients achieved clinical remission off and on GC, respectively. None of the patients achieved complete clinical and serologic complete remission. Patients achieving LLDA had an older age of onset compared to those with higher disease activity $(\mathrm{p}=0.003)$, and a lower prevalence of fever $(p=0.009)$, weight loss $(p=0.07)$, cutaneous vasculitis $(p=0.002)$, serositis $(p=0.006)$, nephritis $(p=0.02)$, a lower median SDI score, and lower prevalence of developing severe damage (SDI $\geq 3)(p=0.04)$. Predictors of LLDA were an older age of onset $[p=0.006(\mathrm{OR}=1.05 ; 95 \% \mathrm{CI}=1.01-1.09)]$ and weight loss $[p=0.009(\mathrm{OR}=5$; 95\% CI=1.9-16.5)]; whereas patients with LLDA were less likely to have cutaneous vasculitis $[p=0.01(\mathrm{OR}=0.2 ; 95 \% \mathrm{CI}=0.06-0.7)]$ or pleurisy and/or pleural effusion $[p=0.001(\mathrm{OR}=0.2 ; 95 \% \mathrm{CI}=0.1-0.5)]$.
\end{abstract}

Correspondence to: Dr. Basma M. Medhat, E-Mail: basmamedhat@kasrlainy.edu.eg
Conclusion: Achieving remission was substantially low. Lupus Low Disease Activity (29.6\%) was associated with a higher age of onset, several distinct clinical characteristics, and lower damage.

Key Words: Systemic lupus erythematosus - Lupus low disease activity - Disease activity - Disease damage.

\section{Introduction}

SEVERAL interrelated factors contribute to the outcome of systemic lupus erythematosus, including disease activity. Furthermore, accrual damage has been associated with high disease activity; hence making remission or low disease activity alluring targets [1].

Interestingly, distinct definitions of remission [2] and Lupus Low Disease Activity (LLDA) [4] have been proposed. Moreover, a 'Treat to Target' approach has been delineated [3]; yet, abiding by it and attempting to achieve the aforementioned targets is quite challenging owing to several factors, including the variation in the disease characteristics, and race and ethnicity [5]; however, studies utilizing the proposed definitions of remission and LLDA as primary end points in the Middle East are lacking to the best of our knowledge.

We aimed in this study to portray the characteristics of patients with LLDA and potential factors influencing disease activity and damage.

\section{Patients and Methods}

The medical records of 243 patients fulfilling the 2012 Systemic Lupus Collaborating Clinics (SLICC) classification criteria for SLE [6] managed at the Rheumatology and Rehabilitation and Internal Medicine Departments of Cairo and Ain Shams Universities from January to December 2019 were viewed. The study was approved by the Local 
Ethics Committee, according to the provisions of the World Medical Association Declaration of Helsinki.

\section{Baseline characteristics:}

Demographic data recorded included gender, the age at the last recorded visit and at the onset, where the disease onset was determined at the onset of the initial manifestation(s). Cumulative clinical characteristics were documented; with secondary antiphospholipid syndrome being diagnosed according to the modified Sapporo criteria [7]. Serologic markers recorded included antinuclear antibody (ANA), anti-double stranded deoxyribonucleicacid (anti ds-DNA), and complement 3 and 4 were recorded as consumed or normal.

\section{Disease activity and damage:}

Disease activity at onset and last visit was investigated utilizing the Systemic Lupus Erythematosus Disease Activity Index-2K (SLEDAI$2 \mathrm{~K})[8]$; whereas disease damage was assessed through the Systemic Lupus International Collaborating Clinics/American College of Rheumatology Damage Index (SDI) [9], and was recorded as continuous numerical score and dichotomously to assess the prevalence of development of any (SDI $>$ 1) and severe damage ( $\mathrm{SDI}>3$ ) [10]

\section{Definition of remission:}

Remission was determined according to the 'Definition of Remission in SLE project' (DORIS) [2] and Zen et al's proposed definitions [11] ; with the latter study further coupling the definition of remission with the nature of treatment implemented: (i) Complete remission which is a SLEDAI-2K score of zero in the absence of GC intake and the possibility of antimalarial administration only as an immunomodulatory; (ii) Clinical remission in absence of GC implementation: Defined as SKLEDAI-2K score of $<4$, with the presence of serologic activity in the form of hypocomplementinemia and/or positive anti-ds DNA; yet with patients being off $\mathrm{GC}$ and antimalarials and other immunosuppresives are administered; (iii) Clinical remission: A SLEDAI-2K score of $<4$ and serologic activity in the form of hypocomplementinemia and/or positive anti-ds DNA, and patients are receiving $\mathrm{GC}$ in a dose equivalent to prednisolone $5 \mathrm{mg} /$ day.

\section{Definition of lupus low disease activity state:}

Lupus Low Disease Activity state was defined as a SLEDAI-2K $<4$ in the absence of major organ involvement (fever, hemolytic anemia, central nervous system, renal involvement, cardiopulmonary, and vasculitis), GC dosage $<7.5 \mathrm{mg} /$ day, no evidence of new activity compared to the prior visit, well tolerated of immunosuppressive or biologic agents, and a physician global assessment of $<1$ (on a scale of 0-3) [4].

\section{Statistical method:}

Data were coded and entered using the statistical package for the Social Sciences (SPSS) version 26 (IBM Corp., Armonk, NY, USA). Data was summarized using median and interquartile range in quantitative data and using frequency (count) and relative frequency (percentage) for categorical data. Comparisons between quantitative variables were done using the non-parametric Mann-Whitney test. For comparing categorical data, Chi square $\left(\xi^{2}\right)$ test was performed. Exact test was used instead when the expected frequency is less than 5. Logistic regression was done to detect independent predictors of LLDA. $p$-values less than 0.05 were considered as statistically significant.

\section{Results}

\section{Baseline characteristics:}

This retrospective study included 243 patients, including $33(13.6 \%)$ males and $210(86.4 \%) \mathrm{fe}$ males. The mean age at the last visit and at onset was 31.4 (9.4) and 24.5 (8.5) years, respectively; with the median disease duration being 72 (Interquartile Range (IQR): 44-120)]. Mucocutaneous manifestations were the most prevalent being present in 192 (79\%) patients, followed by arthritis which was present in $170(70 \%)$ patients. Other cumulative clinical and serologic characteristics are shown in Table (1).

Table (1): Baseline cumulative clinical and serologic characteristics of the cohort*.

\begin{tabular}{ll}
\hline Cumulative clinical characteristics & $\mathrm{N}=243$ \\
\hline Constitutional & $110(45.3)$ \\
Mucocutaneous & $192(79)$ \\
Arthritis & $170(70)$ \\
Serositis & $86(35.4)$ \\
Hematologic & $147(60.5)$ \\
Nephritis & $139(57.2)$ \\
Neuropsychiatric & $80(32.9)$ \\
APS & $42(17.3)$ \\
Serologic characteristics: & \\
ANA & $239(98.4)$ \\
Anti-ds DNA & $150(61.7)$ \\
$\quad$ aPpocomplementinemia & $159(65.4)$ \\
Comorbidities & $81(33.3)$ \\
\hline
\end{tabular}

*Unless indicated, data is presented in number and percentage. Abbreviations:

APS : Antiphospholipid syndrome.

ANA: Anti-nuclear antibody.

Anti-ds DNA: Anti-double stranded deoxyribonucleic acid antibody. aPL : Antiphospholipid antibodies. 


\section{Disease activity and damage:}

The definition of LLDA was met in about third of our patients [72/243 (29.6\%)]. On the other hand, the majority of our cohort [142/243 (58.4\%)] had a SLEDAI-2K score $>4$ and/or received a GC dosage of $>7.5 \mathrm{mg}$ was; while $1(0.4 \%)$ and 28 (11.5\%) patients achieved clinical remission off and on GC, respectively. None of the patients achieved complete clinical and serologic complete remission (Table 2).

Table (2): Disease activity and damage of the cohort (243 patients)

\begin{tabular}{ll}
\hline Disease activity: & \\
SLEDAI-2K & Median (IQR) \\
At onset & $12(8-22)$ \\
At the last visit & $4(1-8)$ \\
State of remission achieved: & $\mathrm{N}(\%)$ \\
Complete with serologic activity (Off GC) & $1(0.4)$ \\
Partial remission (on GC) & $28(11.5)$ \\
LLDA with GC dose $\leq 7.5 m g / d a y$ & $72(29.6)$ \\
SLEDAI-2K >4 and/or GC dose & $142(58.4)$ \\
>7.5mg/day & \\
Disease damage: & \\
SDI score at last visit [Median (IQR)] & $1(0-3)$ \\
Damage attained: & $\mathrm{N}(\%)$ \\
Any damage (SDI $\geq 1)$ & $178(73.3)$ \\
Severe damage (SDI $\geq 3)$ & $73(30)$ \\
\hline
\end{tabular}

*Unless indicated, data is presented in number and percentage.

Abbreviations:

IQR : Interquartile range.

- Systemic Lupus Erythematosus Disease Activity Index-2K (SLEDAI2K).

GC : Glucocorticoids.

LLDA: Lupus Low Disease Activity.

SDI : Systemic Lupus International Collaborating Clinics/American College of Rheumatology Damage Index

\section{Characteristics of patients with LLDA:}

Of the patients with LLDA [72/243 (29.6\%) patients], $8 / 72(11.1 \%)$ were males. The mean age at the last visit of this group of patients was 33.7 (10.3) years, whereas the mean age at onset was $26.6( \pm 9.4)$ years. The mean disease duration was $86( \pm 48)$ months. Cumulative clinical, serologic characteristics of patients with LLDA and the association of LLDA with disease damage are shown in Table (3).

Differences between patients with LLDA and SLEDAI-2K > 4:

The low prevalence of patients achieving remission hampered the analysis of these subgroups. On the other hand, upon assessing patients with LLDA, there were several differences between them and those who had at last visit a SLEDAI$2 \mathrm{~K}>4$ and/or were receiving a GC dose of $>7.5 \mathrm{mg} /$ day at the last visit. Of the demographic features recorded, patients characterized by having a low activity state had an older age of onset ( $p=0.003)$; yet there was no gender differences between the two groups $(p=0.5)$ and no association with the disease duration $(p=0.4)$ (Table 4$)$.

Table (3): Baseline cumulative clinical, and serologic characteristics of patients achieving Lupus Low Disease Activity at the last visit*.

\begin{tabular}{ll}
\hline Cumulative clinical characteristics & $\mathrm{N}=72$ \\
\hline Constitutional & $28(38.9)$ \\
Mucocutaneous & $53(73.9)$ \\
Arthritis & $51(70.8)$ \\
Serositis & $18(25)$ \\
Hematologic & $44(61.1)$ \\
Nephritis & $36(50)$ \\
Neuropsychiatric & $18(25)$ \\
APS & $15(20.8)$ \\
Serologic characteristics: & \\
$\quad$ ANA 71 & $71(98.6)$ \\
$\quad$ Anti-ds DNA & $41(56.9)$ \\
$\quad$ Hypocomplementinemia & $43(59.7)$ \\
aPL & $23(31.9)$ \\
Comorbidities & $29(40.3)$ \\
\hline
\end{tabular}

*Unless indicated, data is presented in number and percentage. Abbreviations:

APS : Antiphospholipid syndrome.

ANA: Anti-nuclear antibody.

Anti-ds DNA: Anti-double stranded deoxyribonucleic acid antibody. aPL : Antiphospholipid antibodies.

Table (4): Demographic differences between patients with achieving LLDA and those with a SLEDAI-2K >4 and/or receiving GC $>7.5 \mathrm{mg} /$ day $I$ I.

\begin{tabular}{llll}
\hline & $\begin{array}{c}\text { LLDA } \\
\mathrm{N}=72\end{array}$ & $\begin{array}{c}\text { SLEDAI-2K >4 or } \\
\text { GC dose }>7.5 \\
\mathrm{~N}=142\end{array}$ & $\begin{array}{c}p- \\
\text { value* }\end{array}$ \\
\hline $\begin{array}{l}\text { Demographic } \\
\text { characteristics: }\end{array}$ & & & \\
$\begin{array}{l}\text { Age (years) } \\
\text { [Median (IQR)]: }\end{array}$ & & & \\
Age at onset & $25(19-33)$ & $20(17-27)$ & 0.003 \\
Age at last visit & $32(26.5-39)$ & $28(23-35)$ & 0.006 \\
Gender: & & & \\
Males & $8(11.1)$ & $20(14.1)$ & \\
Females & $64(88.9)$ & $122(85.9)$ & 0.5 \\
Disease duration & $84(48-120)$ & $72(48-120)$ & 0.4 \\
(months) & & & \\
[Median (IQR)] & & & \\
\hline
\end{tabular}

IUUnless indicated, data is presented in number and percentage. $*$ Significant $p$-value $<0.05$.

Abbreviations:

LLDA: Lupus Low Disease Activity.

GC : Glucocorticoids.

IQR : Interquartile range.

Among the cumulative clinical characteristics investigated, patients with LLDA had a lower prevalence of fever $(p=0.009)$ and weight loss 
$(p=0.07)$. Moreover, they showed a lower prevalence of cutaneous vasculitis $(p=0.002)$, serositis $(p=0.006)$, and nephritis $(p=0.02)$. On the other hand, there was no difference between the two groups in any of the studied serologic markers. It is of note that patients having a lower SLEDAI$2 \mathrm{~K}$ at onset were more inclined to achieve LLDA $(p<0.001)$ (Table 5).

Table (5): Comparison between patients achieving LLDA and those with a SLEDAI-2K >4 and/or receiving GC $>7.5 \mathrm{mg} /$ daygI.

\begin{tabular}{|c|c|c|c|}
\hline & $\begin{array}{l}\text { LLDA } \\
\mathrm{N}=72\end{array}$ & $\begin{array}{c}\text { SLEDAI-2K }>4 \text { or GC dose }>7.5 \\
\mathrm{~N}=142\end{array}$ & $\begin{array}{c}p- \\
\text { value* }\end{array}$ \\
\hline \multicolumn{4}{|l|}{ Cumulative clinical characteristics: } \\
\hline Constitutional & $28(38.9)$ & $75(52.8)$ & 0.05 \\
\hline Fever & $24(33.3)$ & $74(52.1)$ & 0.009 \\
\hline Lymphadenopathy & $3(4.2)$ & 0 & 0.03 \\
\hline Significant unintended weight loss & $9(12.5)$ & $8(5.6)$ & 0.07 \\
\hline Mucocutanoeus & $54(73.6)$ & $118(83.1)$ & 0.1 \\
\hline Cutaneous vasculitis & $3(4.2)$ & $29(20.4)$ & 0.002 \\
\hline Malar rash & $44(61.1)$ & $100(70.4)$ & 0.1 \\
\hline Photosensitvity & $22(30.6)$ & $61(43)$ & 0.1 \\
\hline Discoid rash & $3(4.2)$ & $11(7.7)$ & 0.3 \\
\hline Oral ulcers & $18(25)$ & $51(35.9)$ & 0.1 \\
\hline Alopecia & $31(43.1)$ & $63(44.4)$ & 0.8 \\
\hline Arthritis & $51(70.8)$ & $104(73.2)$ & 0.7 \\
\hline Serositis & $18(25)$ & $63(44.4)$ & 0.006 \\
\hline Pleurisy and/or pleural effusion & $14(19.4)$ & $56(39.4)$ & 0.003 \\
\hline Pericarditis and/or pericardial effusion & $8(11.1)$ & $17(12)$ & 0.8 \\
\hline Nephritis & $36(50)$ & $93(65.5)$ & 0.02 \\
\hline Neuropsychiatric & $18(25)$ & $54(38)$ & 0.05 \\
\hline Psychosis & $4(5.6)$ & $19(13.4)$ & 0.08 \\
\hline Seizures & $4(5.6)$ & $9(6.3)$ & 1 \\
\hline Peripheral and/or cranial neuropathy & $3(4.2)$ & $8(5.6)$ & 0.7 \\
\hline Hematologic & $44(61.1)$ & $84(59.2)$ & 0.7 \\
\hline Hemolytic anemia & $8(11.1)$ & $23(16.2)$ & 0.3 \\
\hline Thrombocytopenia & $9(12.5)$ & $31(21.8)$ & 0.09 \\
\hline Leukopenia & $41(56.9)$ & $73(51.4)$ & 0.4 \\
\hline Secondary antiphospholipid syndrome & $15(20.8)$ & $24(16.9)$ & 0.4 \\
\hline Associated comorbidities & $29(40.3)$ & $80(56.3)$ & 0.02 \\
\hline \multicolumn{4}{|l|}{ Serologic: } \\
\hline ANA & $71(98.6)$ & $139(97.9)$ & 1 \\
\hline Anti-ds DNA & $41(56.9)$ & $90(63.4)$ & 0.3 \\
\hline Hypocomplementinemia & $43(59.7)$ & $98(69)$ & 0.1 \\
\hline $\mathrm{aPL}$ & $23(31.9)$ & $52(36.6)$ & 0.4 \\
\hline SLEDAI at onset [Median (IQR)] & $10(8-16)$ & $16(10-25)$ & $<0.001$ \\
\hline \multicolumn{4}{|l|}{ Disease damage: } \\
\hline SDI score [Median (IQR)] & $1(0-2)$ & $2(1-3)$ & 0.008 \\
\hline Presence of any damage (SDI > 1 ) & $49(68.1)$ & $111(78.2)$ & 0.1 \\
\hline Presence of severe damage (SDI $>3$ ) & $17(23.6)$ & $53(37.3)$ & 0.04 \\
\hline
\end{tabular}

IIUnless indicated, data is presented in number and percentage. $*$ Significant $p$-value $<0.05$.

Abbreviations:

LLDA : Lupus Low Disease Activity.

GC : Glucocorticoids.

IQR : Interquartile range.

APS : Antiphospholipid syndrome.

ANA : Anti-nuclear antibody.

Anti-ds DNA: Anti-double stranded deoxyribonucleic acid antibody.

aPL : Antiphospholipid antibodies.

SDI : Systemic Lupus International Collaborating Clinics/American College of Rheumatology Damage Index 
Association of disease activity with accrual damage:

The median SDI score was higher among patients with higher disease activity $(p=0.008)$. Although there was no difference in the prevalence of attaining any damage (SDI > 1 ) between the two groups $(p=0.1)$, the prevalence of developing severe damage (SDI > 3) was higher among patients with a higher SLEDAI-2K $(p=0.04)$.

\section{Predictors of LLDA:}

Upon multivariate logistic regression analysis, patients with LLDA were more inclined to have an older age of onset $[p=0.006(\mathrm{OR}=1.05 ; 95 \%$ $\mathrm{CI}=1.01-1.09)]$ and manifest with weight loss $[p=0.009(\mathrm{OR}=5 ; 95 \% \mathrm{CI}=1.9-16.5)]$. On the other hand, they were less likely to develop cutaneous vasculitis $[p=0.01(\mathrm{OR}=0.2 ; 95 \% \mathrm{CI}=0.06-0.7)]$ or pleurisy and/or pleural effusion [ $p=0.001$ $(\mathrm{OR}=0.2 ; 95 \% \mathrm{CI}=0.1-0.5)]$.

\section{Discussion}

The management of SLE is challenging owing to several factors; including the racial, ethnic, and individual variations characterizing the disease nature [12]. Hence, the emerging concept of 'Treat to Target', although described in SLE [3], is seldom achieved, unlike other rheumatic diseases such as rheumatoid arthritis [13].

Moreover, the variation in the proposed definitions of SLE activity and remission across various studies could be considered as one of the main factors adding to the disparity in the prevalence of remission and disease activity states achieved $[2,11,14-19]$. In our study, remission was determined according to that defined by the DORIS project [2] and Zen et al. [11], and LLDA was defined according to that described by Franklyn et al. [4]; with the prevalence of LLDA at the last visit being about $30 \%$ and that of clinical remission off and on GC being $0.4 \%$ and $2811.5 \%$, respectively. On the other hand, none of the patients achieved complete serologic and clinical remission. In a previous study from the Netherlands [20], the prevalence of achieving LLDA was $76 \%$; yet this prevalence represented the occurrence of LLDA at least once throughout the course of the disease; whereas the prevalence of LLDA in a previous multiethnic cohort was 44\% [12]. Interestingly, a previous report demonstrated a higher prevalence of remission achieved on (20.1\%) and off GC (12.9\%) [21] as compared to that detected in our study. The variation in the prevalence of remission and LLDA reported could be attributed to several factors including the implemented definition of low activity and remission, timing of assessment, and study design.

Interestingly, patients achieving LLDA (29.6\%) showed several differences to those whom failed to achieve either LLDA or remission $(58.4 \%)$. Among the demographic features investigated, patients with LLDA were more inclined to have an older age of onset ( $p=0.003)$. Contrary to our finding, a previous study showed no association of LLDA with the age of onset was; yet, similar to our study, the authors did not detect gender differences or an association with the duration of the disease [21].

Of the cumulative clinical characteristics included, patients with LLDA demonstrated a lower prevalence of fever ( $p=0.009)$, weight loss $(p=0.07)$, cutaneous vasculitis $(p=0.002)$, serositis $(p=0.006)$, and nephritis $(p=0.02)$. Similar to our findings, the presence of renal involvement at baseline and one year follow-up was lower among patients with LLDA in a previous study [21]; yet, unlike our study, the authors detected a higher prevalence of serositis among their patients with LLDA and a lower prevalence of hematologic involvement, which was comparable between both groups in our study $(p=0.7)$.

It is of interest that patients having a lower SLEDAI-2K at onset were more inclined to achieve a low activity state by the last visit $(p<0.001)$. This finding is similar to previous reports [20-22].

The importance of assessing activity in SLE and thriving to achieve it lowest possible state rises from its potential impact on disease damage, as demonstrated in several previous studies [2026]. Similarly, the median SDI score at the last visit was lower among patients with LLDA ( $p=0.008)$; whom developed severe damage less frequently than those demonstrating a higher disease activity $(p=0.04)$.

Predictors of LLDA in our study were an older age of onset $[\mathrm{p}=0.006(\mathrm{OR}=1.05 ; 95 \% \mathrm{CI}=1.01$ 1.09)] and manifesting with weight loss $[\mathrm{p}=0.009$ $(\mathrm{OR}=5 ; 95 \% \mathrm{CI}=1.9-16.5)]$. On the other hand, patients with LLDA were less likely to have cutaneous vasculitis $[\mathrm{p}=0.01(\mathrm{OR}=0.2 ; 95 \% \mathrm{CI}=0.06$ $0.7)]$ and pleurisy and/or pleural effusion $[\mathrm{p}=0.001$ $(\mathrm{OR}=0.2 ; 95 \% \mathrm{CI}=0.1-0.5)]$. Interestingly, a previous study [12] assessing the predictors of LLDA demonstrated that LLDA was associated with an older age of onset, hence similar to our study; yet the authors further detected an association with the disease duration being longer among patients achieving LLDA; whereas there was no association 
with the disease duration in our study. Moreover, in the authors detected that patients with nephritis and discoid rash were less likely to achieve LLDA; whereas the presence of arthritis was associated with achieving LLDA; hence detecting different predictors than those in our study. This disparity could be explained by several factors including race and ethnicity, time of assessment, and patients' inclusion criteria.

Our study's main limitation lies in its retrospective nature that has led to the absence of some data. Yet, it has several strengths; including the participation of two tertiary centers in Egypt and that it has assessed the prevalence of remission and LLDA utilizing distinct definitions; an assessment that to the best of our knowledge has not been investigated in Egypt previously.

To conclude, LLDA was achieved in $29.6 \%$ patients, and was associated with an older age of onset, lower disease activity at baseline, several distinct clinical characteristics. Moreover, disease damage was lower among patients with LLDA.

\section{References}

1- ROMO-TENA J., REYNA-DE LA GARZA, BARTNICKINAVARRETE I., et al.: Factors associated with remission in patients with systemic lupus erythematosus: New insights into a desirable state. Clinical Rheumatology. 37: 3033-42, 2018.

2- VAN VOLLENHOVEN R., VOSKUYL A., BERTSIAS G., et al.: A framework for remission in SLE: consensus findings from a large international task force on definitions of remission in SLE (DORIS). Annals of the Rheumatic Diseases, 76: 554-61, 2017.

3- VAN VOLLENHOVEN R, MOSCA M., BERTSIAS G., et al.: Treat-to-target in systemic lupus erythematosus: recommendations from an international task force. Ann. Rheum. Dis., 73: 958-67, 2014.

4- FRANKLYN K., LAU C.S., NAVARRA S.V., et al.: Definition and initial validation of a lupus low disease activity state (LLDAS). Annals of the Rheumatic Diseases, 75: 1615-21, 2016.

5- GONZALEZ L.A., TOLOZA S.M., MCGWIN Jr. G., et al.: Ethnicity in systemic lupus erythematosus (SLE): Its influence on susceptibility and outcomes. Lupus, 22: 1214-24, 2013.

6- PETRI M., ORBAI A.M., ALARCO N.G.S., et al.: Derivation and validation of the Systemic Lupus International Collaborating Clinics classification criteria for systemic lupus erythematosus. Arthritis Rheum., 64: 2677-86, 2012.

7- MIYAKIS S., LOCKSHIN M.D., ATSUMI T., et al.: International consensus statement on an update of the classification criteria for definite antiphospholipid syndrome (APS). J. Thromb. Haemos., 4: 295-306, 2006.

8- GLADMAN D.D., IBANEZ D. and UROWITZ M.B.: Systemic lupus erythematosus disease activity index. J. Rheumatol., 29: 288-91, 2002.
9- GLADMAN D., GINZLER E., GOLDSMITH C., et al.: The development and initial validation of the Systemic Lupus International Collaborating Clinics/American College of Rheumatology damage index for systemic lupus erythematosus. Arthritis Rheum., 39: 363-9, 1996.

10- LOPEZ R., DAVIDSON J.E., BEEBY M.D., et al.: Lupus disease activity and the risk of subsequent organ damage and mortality in a large lupus cohort. Rheumatology, 51: 491-8, 2012.

11- ZEN M., IACCARINO L., GATTO M., et al.: Prolonged remission in Caucasian patients with SLE: Prevalence and outcomes. Annals of the rheumatic diseases, 74: 2117 22, 2015.

12- GOLDER V., KANDANE-RATHNAYAKE R., HOI A.Y., et al.: Frequency and predictors of the lupus low disease activity state in a multi-national and multi-ethnic cohort. Arthritis Research \& Therapy, 18: 260, 2016.

13- RÍOS-GARCÉS R., ESPINOSA G., VAN VOLLENHOVEN R., et al.: Treat-to-target in systemic lupus erythematosus: Where are we? European Journal of Internal Medicine. Feb 1, 2020.

14- NOSSENT J., KISS E., ROZMAN B., et al.: Disease activity and damage accrual during the early disease course in a multinational inception cohort of patients with systemic lupus erythematosus. Lupus, 19: 949-56, 2010.

15-ZEN M., BASSI N., NALOTTO L., et al.: Disease activity patterns in monocentric cohort of SLE patients: A sevenyear follow-up study. Clin. Exp. Rheumatol., 30: 856-63, 2012.

16- BARR S.G., ZONANA-NACACH A., MAGDER L.S., et al.: Patterns of disease activity in systemic lupus erythematosus. Arthritis Rheum., 42: 2682-8, 1999.

17- LAUSTRUP H., VOSS A., GREEN A., et al.: SLE disease patterns in a Danish population-based lupus cohort: An 8-year prospective study. Lupus, 19: 239-46, 2010.

18- HELLER C.A. and SCHUR P.H.: Serological and clinical remission in systemic lupus erythematosus. J. Rheumatol., 12: 916-18, 1985.

19- WALZ LEBLANC B.A.E., GLADMAN D.D. and UROWITZ M.B.: Serologically active clinically quiescent systemic lupus erythematosus-predictors of clinical flares. J. Rheumatol., 2: 2239-41, 1994.

20- TSANG-A-SJOE M.W., BULTINK I.E., HESLINGA M. et al.: Both prolonged remission and Lupus Low Disease Activity State are associated with reduced damage accrual in systemic lupus erythematosus. Rheumatology, 31 : kew377. 2016.

21- ROMO-TENA J., REYNA-DE LA GARZA R., BARTNICKI-NAVARRETE I., ALCOCER-VARELA J. and GÓMEZ-MARTIN D.: Factors associated with remission in patients with systemic lupus erythematosus: New insights into a desirable state. Clinical Rheumatology, 37: 3033-42, 2018.

22- TANI C., VAGELLI R. and STAGNARO C.: Remission and low disease activity in systemic lupus erythematosus: an achievable goal even with fewer steroids? Real-life data from a monocentric cohort. Lupus Science \& Medicine, 5, 2018.

23- ZEN M., IACCARINO L., GATTO M., et al.: Lupus low disease activity state is associated with a decrease in 
damage progression in Caucasian patients with SLE, but overlaps with remission. Annals of the Rheumatic Diseases, 77: 104-10, 2018.

24- PETRI M., PURVEY S., FANG H., et al.: Predictors of organ damage in systemic lupus erythematosus: The Hopkins Lupus Cohort. Arthritis \& Rheumatism, 64: 4021-8, 2012.

25- NOSSENT J., KISS E., ROZMAN B., et al.: Disease activity and damage accrual during the early disease course in a multinational inception cohort of patients with systemic lupus erythematosus, 19: 949-56, 2010.

26- SHARMA C., RAYMOND W., EILERTSEN G., et al.: Achieving Lupus Low Disease Activity State (LLDAS50 ) is associated with both reduced damage accrual and mortality in patients with Systemic Lupus Erythematosus. Arthritis Care \& Research, 1, 2019.

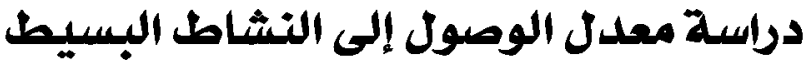

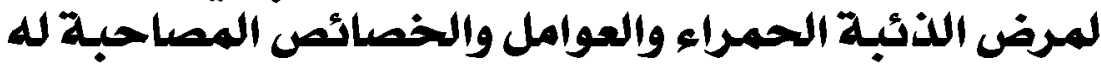

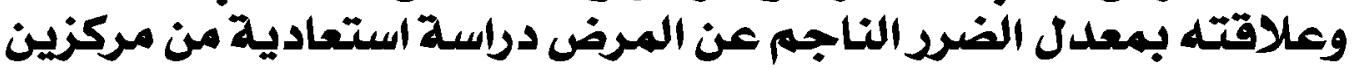

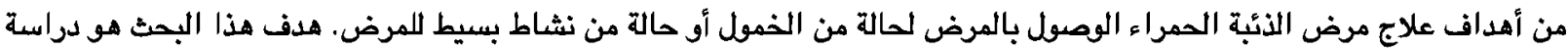

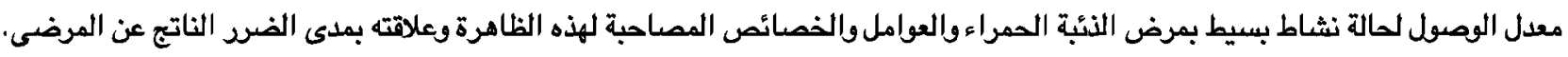

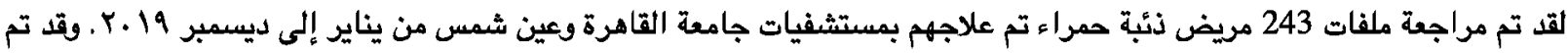

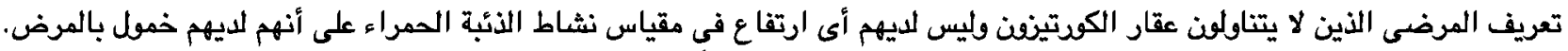

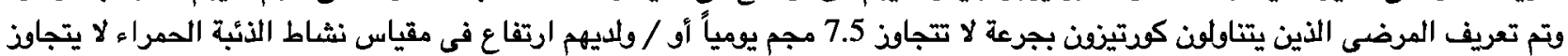

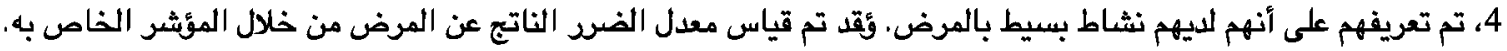

ؤقد تبين أن (58.4\%) 142 مريضاً كان لديهم نشاط عالى بالمرض حيث أنهم كانوا يتناولون جرعات كورتينون تتجاوذ الـ 7.5 مجم فى في

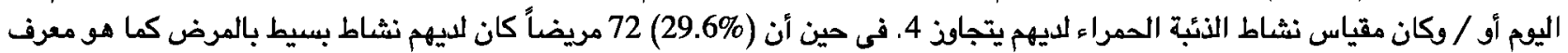

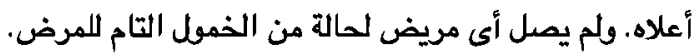

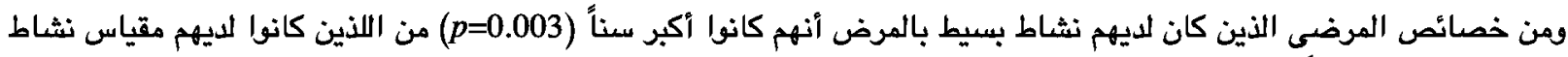

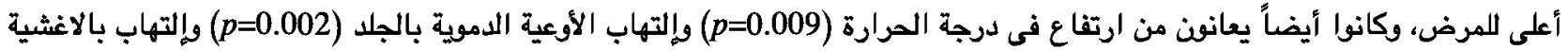

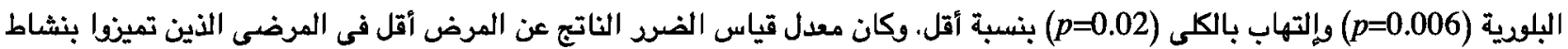

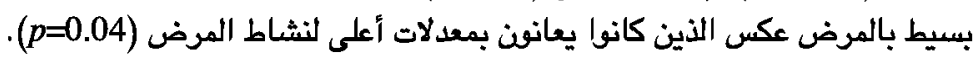

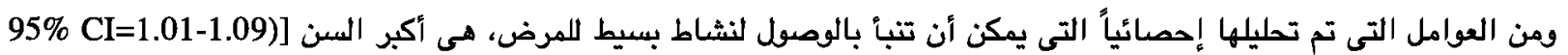

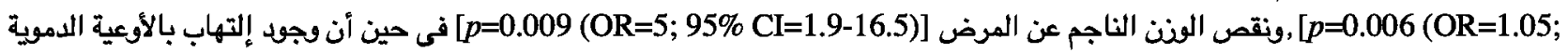

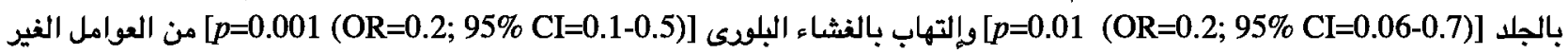
مصاحبة لحالة النثاط البسيط المرض. 